\title{
Pre-pulseless Takayasu Arteritis Presenting with Hypertension
}

\author{
SMS ISLAM ${ }^{\mathrm{a}}, \mathrm{S} \mathrm{NASRIN}^{\mathrm{b}}$, FACADER $^{\mathrm{c}}$, MM HAQ $^{\mathrm{d}}$, SDM TAIMUR $^{\mathrm{e}}$
}

\begin{abstract}
Summary:
Takayasu arteritis (TA) is a chronic granulomatous vasculitic disease of the aorta and its major branches, predominantly affecting young females of Asian origin. Also known as Aortic arch syndrome, the condition has been better documented as the 'Pulse less disease' leading to claudication, bruits, absent or reduced brachial or radial pulses and aortic incompetence.
\end{abstract}

However, a 'prepulseless'stage of TA exists, characterized by non-specific constitutional symptoms, hypertension and

\section{Introduction:}

Takayasu arteritis (TA) is a chronic inflammatory arteritis affecting large vessels, predominantly the aorta and its main branches and the pulmonary artery. ${ }^{1}$ It is rare, but most commonly seen in Japan, South East Asia, India, and Mexico. ${ }^{2,3}$ The disease commonly presents in the 2nd or 3rd decade of life, ${ }^{3,4}$ and primarily affects young females, with the female to male ratio declining from Eastern Asia towards the West. ${ }^{2,3}$

The clinical course of TA is characterized by two often overlapping phases, the acute, active inflammatory prepulseless phase and the chronic pulseless end-stage

a. Dr. S.M. Shaheedul Islam, Associate Professor \& Consultant in Paediatric Cardiology, Ibrahim Cardiac Hospital \& Research Institute (ICHRI), Dhaka. Email : shaheedul.islam@yahoo.com

b. Dr. Sahela Nasrin, Associate Consultant \& Assistant Professor, ICHRI, Dhaka.

c. Dr. F. Aaysha Cader, Resident in Cardiology, ICHRI, Dhaka.

d. Prof. M. Maksumul Haq, Professor \& Head, Department of Cardiology, ICHRI, Dhaka.

e. Syed Dawood Md. Taimur, Associate Consultant \& Assistant Professor, ICHRI, Dhaka.

Address of Correspondence: Dr. S.M.Shaheedul Islam, Associate Professor \& Consultant in Pediatric Cardiology, Ibrahim Cardiac Hospital \& Research Institute (ICHRI), Dhaka. Email :shaheedul.islam@yahoo.com. Tel No.01711362927 / 01749419893

Received: 20 Nov. 2015

Accepted: 25 December 2016 raised inflammatory markers. We report the case of an 18 year-old Bangladeshi female presenting with hypertension, moderate to severe aortic regurgitation (AR) and fever, diagnosed and treated as a case of pre-pulseless TA.

Key words :Takayasu arteritis. Pre-pulseless phase.Aortic regurgitation. Hypertension.

(J Bangladesh Coll Phys Surg 2017; 35: 91-96)

phase. ${ }^{1,2}$ The diagnosis of TA remains a challenge, especially in the pre-pulseless phase, and there is often a delay in diagnosis especially among juveniles. ${ }^{3}$

The early "pre-pulseless" phase is characterised by non-specific features - such as hypertension, headache, fever, muscle pain, arthralgia, myalgia, night sweats, weight loss, abdominal pain, nausea and vomiting, mild anaemia and elevated ESR, followed by a chronic "pulseless" phase, in some cases accompanied by intermittent flares ${ }^{2,4-7}$. We report here the case of an 18year-old Bangladeshi female presenting with hypertension, fever, easy fatiguability, anemia and moderate to severe aortic regurgitation (AR), who was diagnosed as a case of pre-pulseless TA.

\section{Case report:}

An 18-year-old Bangladeshi female presented with the complaints of fever, sore neck, headache, giddiness and backache of three months duration. She married recently and was hypertensive for 3 years. The headache was frontal, throbbing in nature, and associated with occasional nausea. She also had chest pain, more marked during exertion, easy fatiguabilityand large joint arthralgia. She was intermittently febrile, which was low-grade, associated with night sweats but denied any contact with Tuberculosis (TB) patients. There was no history of skin rash, claudication or colour changes on cold exposure.

On physical examination, she was moderately pale. Both radial pulses were palpable, regular, 86 beats/min, high 
volume, collapsing and apparently symmetrical on both sides, with no radio-femoral delay. Femoral and other lower limb pulses were present and of normal volume; pistol shot sound was audible over the femoral arteries. She had dancing carotid pulsations, and Quincke's sign was positive. Blood Pressure was 140/85-20mmHg. This expresses that while the systolic BP is $145 \mathrm{mmHg}$, in the diastolic BP, muffling (i.e. Korotkoff phase IV) occurred at $85 \mathrm{mmHg}$ but the sounds continued to be audible until $20 \mathrm{mmHg}$, coinciding with Korotkoff phase $\mathrm{V}$ (phase of silence). Apex beat was thrusting, in the left 5 th intercostal space just lateral to the midclavicular line. Auscultation revealed a to and fro murmur audible due to the huge regurgitation as well as the resulting systolic ejection murmur (similar to that found in aortic stenosis) caused by a large stroke volume ejected with rapid force. A few bi-basal crackles were heard on lung auscultation. Bruits were heard over the carotid artery of left side. All other systemic examinations, including optic fundi revealed no abnormality.

Initial laboratory workup revealed a Hemoglobin of $7.8 \mathrm{~g} /$ dl, MCV-55.1fL, MCH-16pg, Total Count(TC)-11600/mm, Neutrophil- 72\%, Lymphocyte- 20\%, Monocyte- 04\%, Eosinophil-04\%, platelets- 490000. Erythrocyte sedimentation rate (ESR) - $91 \mathrm{~mm}$ in $1^{\text {st }}$ hour. C Reactive Protein(CRP)-96mg/L,ASO titre-1600 IU/ml. Anti-nuclear antibody (ANA) test was negative. Blood glucose, serum electrolytes, liver and renal function tests, TSH, serum cholesterol and urine analysis were normal. 3 successive samples of blood cultures revealed no growth. Rheumatoid factor was $<8 \mathrm{IU} / \mathrm{ml}$. TPHA was negative and VDRL nonreactive. Mantoux test (MT) and QuantiFERON-Gold (QFT$\mathrm{G})$ test for tuberculosis were negative.
Chest X ray revealed cardiomegaly with LV type apex. ECG fulfilled the voltage criteria of left ventricular hypertrophy. Echocardiography revealed moderate to severe AR (grade III), hugely dilated aortic root $(48 \mathrm{~mm})$ with holodiastolic flow reversal at proximal descending aorta. (Figure $1 \&$ 2). LV was dilated; LVIDd 63.3mm (39 $-53 \mathrm{~mm}$ ), LVIDs $41.5 \mathrm{~mm}, \mathrm{LVEF}-52.92 \%$. Aortic valve is tricuspid. Estimated PASP $45 \mathrm{mmHg}$.

Real time duplex carotid imaging revealed increased intima-medial thickness on the left side, with rest of the carotid and vertebral systems being normal. Recent CT scan of the brain revealed no abnormality. Ultrasonogram of whole abdomen revealed no significant abnormality.

She was transfused with 3 units of packed cells. For hypertension, she was started on Losartan Potassium 50mg OD, Metoprolol 25mg BD and Frusemide/ Spironolactone combination which she is currently on, with fair BP control. Having ruled out the differential diagnoses of syphilis, TB and Systemic Lupus Erythematosis (SLE) by negative blood work, we proceeded to a working diagnosis of Rheumatic Fever, given her raised ASO titres, and commenced her on high dose aspirin and oral prednisolone starting from $60 \mathrm{mg}$ OD in a tapering dose along with penicillin. However, she showed no clinical improvement; ASO titre, ESR \& CRP remained persistently high. As she did not fulfil the modified Duckett Jones criteria for rheumatic fever, and also as there were no valvular lesions suggestive of rheumatic carditis on echo, the differential diagnosis of Rheumatic Fever was abandoned. Aspirin and penicillin were omitted. In addition, a relook echo

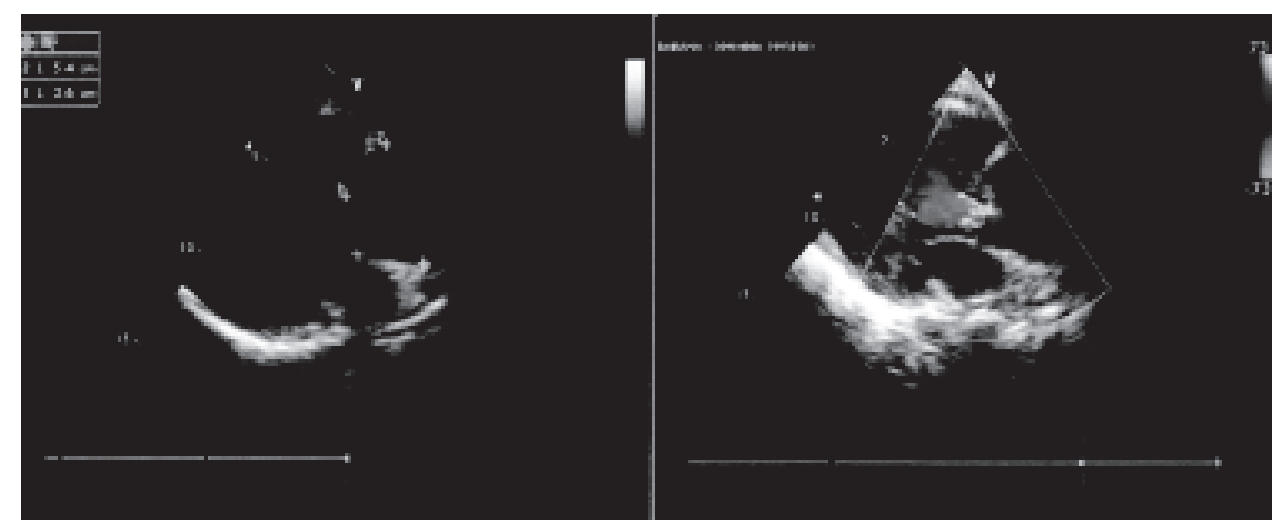

Fig.-1: (a) $2 D$ echocardiography showing dilated aortic root. (b) Colour Doppler echo showing aortic regurgitation 


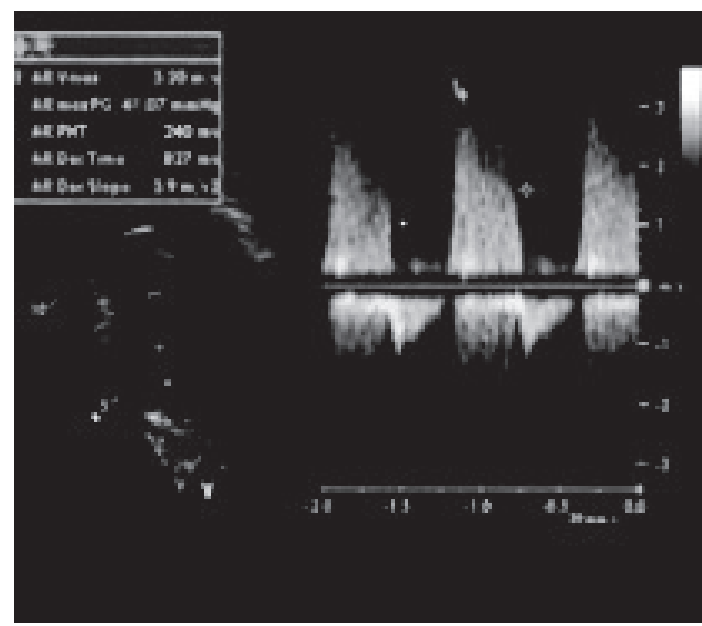

Fig.-2: Continuous wave Doppler echo showing aortic regurgitation AR deceleration velocity

revealed a stenotic lesion in the descending thoracic aorta; the gradient across the stenotic lesion was measured at $62.7 \mathrm{mmHg}$.

We then proceeded to further imaging: a multiple axial CT angiogram of the aorta revealed dilated root of aorta $(49.2 \mathrm{~mm})$ and proximal portion of the ascending aorta $(32.2 \mathrm{~mm})$. Arch of aorta appeared normal. A narrowing was noted in the descending thoracic aorta $(7.71 \mathrm{~mm})$ with post-stenotic dilatation (14.7mm) (Figure 3). A3D MR angiographyconcurred with these findings: moderately dilated ascending aorta more marked in the $\operatorname{root}(49.44 \mathrm{~mm})$, with normal aortic arch and its branches revealing no abnormality (Figure 4).

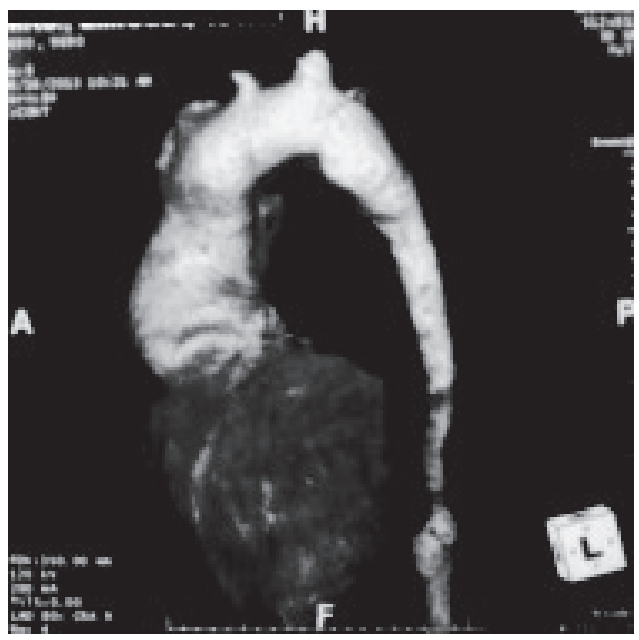

Fig.-3: CT angiogram showing dilated aortic root and stenosis of descending thoracic aorta

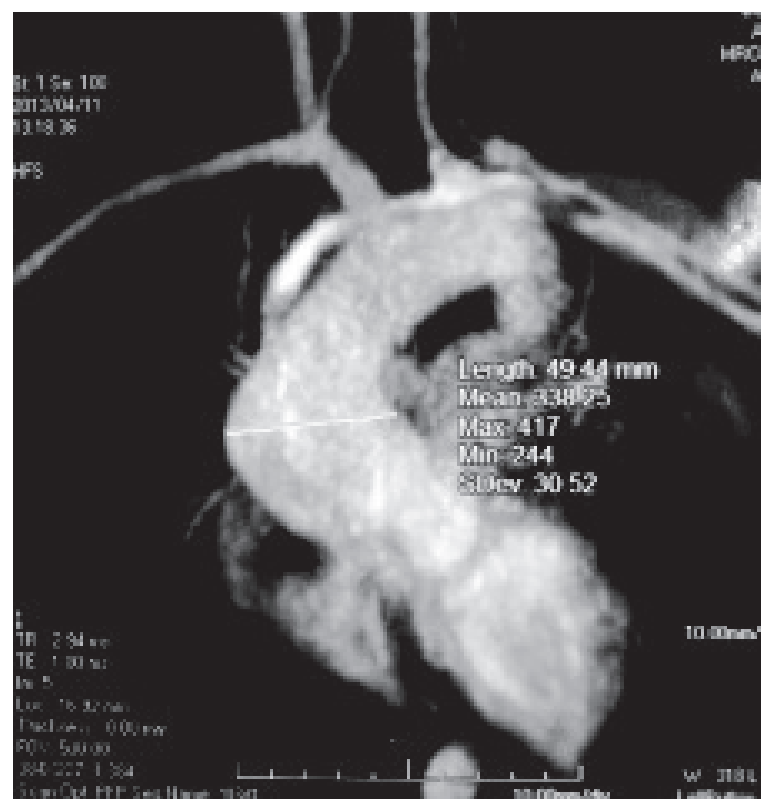

Fig.-4: MRAngiogram showing dilated aortic root, width $49.44 \mathrm{~mm}$.

Simultaneous dilatation and stenotic lesions of the aorta this time, led us to consider vasculitic lesions. As our patient did not fulfill the American College of Rheumatology (ACR) criteria for Takayasu's disease, and as there was no specific criteria for the pre-pulseless phase of TA, we persisted with the latter diagnosis and retained her on high dose steroids, which is the advocated pharmacological treatment for TA. Oral prednisolone was continued for a prolonged period. Following treatment, 6 weeks later, investigations revealed a gradual decline in inflammatory markers : ESR $-25 \mathrm{~mm}$ in $1^{\text {st }}$ hour; CRP $<6 \mathrm{mg}$ / $\mathrm{L}$, ASO titre $800 \mathrm{IU} / \mathrm{ml}$, as well as symptomatic improvement. Impeded by the development of iatrogenic Cushing's syndrome, prednisolone was tapered to a lower maintenance dose, and oral methotrexate was started. Definitive management for the anatomic lesion is surgical, possibly a valve-sparing aortic root reconstruction surgery. Further, a peripheral angiography and concomitant stenting of the stenosis of descending thoracic aorta has been planned, in addition to antiinflammatory medication in the form of prednisolone and cytotoxic drugs with regular monitoring haematological and liver function tests.

\section{Discussion:}

The early "pre-pulseless" (pre-occlusive) phase of TA is of variable severity and duration and its 
manifestations may occur episodically over many years. ${ }^{8}$ Strachan ${ }^{8,9}$ described the full spectrum of pre-pulseless manifestations ofTA. Our patient also showed many of the features Strachan described such asanaemia, prolonged fever, polyarthritis, cranio-cervical pain, vascular bruits, tenderness over the carotid vessels (angiodynia) and elevation of acute phase reactants. The sore neck our patient experienced is attributed to carotitidis, as TA is an inflammatory arteriopathy.

During the early pre-pulseless phase, the peripheral pulses and blood pressure may be normal, making the diagnosis difficult; even aortography may not demonstrate stenosis, occlusion, dilatation or aneurysms ${ }^{1,10,11}$ In our case, her pulses were bilaterally present; however, with a BP of $140 / 85-20 \mathrm{mmHg}$, she had an elevated systolic but low diastolic blood pressure, signifying the effects ofmoderate to severe AR. This expresses that while the systolic BP is $145 \mathrm{mmHg}$, in case of the diastolic BP, muffling (Korotkoff phase IV) occurred at $85 \mathrm{mmHg}$ but the sounds continued to be audible until $20 \mathrm{mmHg}$, coinciding with Korotkoff phase V (phase of silence). In severe AR and hyperkinetic circulatory states, diastolic pressure should be recorded at both Korotkoff phases IV and V. ${ }^{12}$

The hyperkinetic circulatory state of AR also gives rise to the slight 'buzz' to the carotid pulse, clinically manifested as carotid bruits. ${ }^{13}$ Occasionally, a shudder or thrill can be felt in the carotid pulse representing a palpable bruits or transmitted murmur. ${ }^{13}$

$\mathrm{AR}$ is a common phenomenon in TA, and occasionally occurs due to dilatation of the aortic valve ring and ascending aorta, rupture of an aneurysm, ${ }^{14}$ separation of the valve leaflets, and valve thickening. ${ }^{3,4}$ Consequently, although more definitive of pulseless disease, AR may be seen in pre-pulseless TA as well, and the disease must also be considered in instances of isolated aortic incompetence. ${ }^{8}$ The LV systolic dysfunction in this patient could be explained by both the severity of the AR, as well as by the concomitant myocarditis, which occurs in approximately $18 \%$ of patients with TA, leading to LV systolic dysfunction. ${ }^{15}$

With progression into chronic pulseless phase and the development of vascular insufficiency, additional symptoms and signs manifest, such as extremity claudication, bruits, absent or reduced brachial or radial pulses, systolic blood pressure difference $>10 \mathrm{mmHg}$ between arms, decreased femoral pulses, aortic incompetence, renovascular hypertension, CCF, seizures, syncopal episodes, and funduscopic abnormalities including the wreath-like anastomosis surrounding the optic disc, as originally described by Takayasu. ${ }^{1}$

The pathogenesis of TA is characterized by vessel wall inflammation leading to concentric wall thickening, fibrosis, stenosis and thrombus formation. Symptoms reflect end organ ischaemia. More acute inflammation destroys the arterial media leading to aneurysms. ${ }^{16}$ Since large-vessel biopsies are usually not possible and impractical, histological assessment is limited to those cases undergoing revascularisation procedures. The diagnosis of TA is therefore based on clinical and angiographic criteria. ${ }^{17}$ Doppler ultrasound may be a useful non-invasive procedure for assessment of vessel wall inflammation, but angiography remains the goldstandard.

Few cases have been reported thus far on the 'prepulseless' phase. Due to the non-specificity of the symptoms and the absence of specific laboratory parameters, the disease is often unrecognized in this phase. ${ }^{17}$ TA has been diagnosed at the pre-pulseless period after stenotic lesions of the aorta and its branches were detected on imaging, in case reports of two young females presenting with fever of unknown origin. ${ }^{18} \mathrm{An}$ absence of the physical findings related to vasculitic lesions, common in the pre-pulseless period, should not rule out the disease. ${ }^{18}$

The commonest mode of presentation of TA among Indian-origin patients is hypertension (83\%), ${ }^{7}$ along with headache and left ventricular hypertrophy $4,6,7$ as in this case. Her hypertension is explained as occurring as a result of AR, not coarctation, as the stenosis only involved the more distal part of the descending thoracic aorta and not the usual site of coarctation, which is just distal to the left subclavian artery.

The American College of Rheumatology (ACR) defined specific diagnostic criteria for Takayasu's arteritis in 1990. ${ }^{19}$ However, there are no specific diagnostic criteria detailed for the diagnosis of pre-pulseless phase of TA. Also, especially given the acknowledged fact that there are no specific diagnostic tests for TA, making the correct diagnosis depends on clinical features in conjunction with vascular imaging abnormalities. ${ }^{15} \mathrm{On}$ 
the basis of supportive vascular imaging we treated the case as one of pre-pulseless TA.

The differential diagnoses of pre-pulseless TA include other causes of large vessel vasculitis, especially inflammatory aortitis such as syphilis, TB, SLE, rheumatoid arthritis and spondyloarthropathies and developmental abnormalities such as coarctation of the aorta. Most have specific features that enable diagnosis: Syphilis affects an older age group, with calcification, sparing the descending thoracic aorta, and stenoses are not a feature. ${ }^{2,4}$

TB is an important differential and possible aetiological factor of TA, with a possible association between the two being proposed, and documented in a recent case report of a young Bangladeshi female presenting with lymphadenopathy and stenosis of subclavian arteries and descending abdominal aorta ${ }^{20}$. In fact, pre-pulseless TA can present with fever and lymphadenopathy, much like TB. ${ }^{21}$ However, aortitis due to TB tends to cause erosion of the vessel wall with the formation of true or false aneurysms, particularly affecting the descending thoracic and abdominal aorta. ${ }^{2}$ The necessity to exclude TB was therefore paramount and both the Mantoux test and the QFT-G was negative, ruling out both latent TB infection (LTBI) and TB disease.QFT-G has a specificity of $98.1 \%$ and a sensitivity of $89.0 \%$ in one study. In another study, the specificity of QFT-G was 96\%, compared with $49 \%$ for the tuberculin skin test. ${ }^{22}$

The ESR is considered the best available routine laboratory indicator for disease activity of TA in adolescents $^{23-25}$ but may continue to be elevated in disease remission. ${ }^{23-25} \mathrm{CRP}$, increasingly measured as a disease activity marker in TA, appears to correlate well with active TA. Furthermore, there is evidence in the literature that suggests that isolated ASO titres have been found in patients with TA, and is not exclusively associated with Rheumatic fever. ${ }^{26,27}$

The Ishikawa clinical classification of $\mathrm{TA}^{24}$ defined four clinical groups based on the natural history and complications of the disease. The four most important complications defined are Takayasu retinopathy, secondary hypertension, aortic regurgitation, and aneurysm formation. Our case has 3 of the 4 complications described.

Adequate therapy in TA is important to prevent irreversible vessel damage with resulting insufficiency of vital organs. Corticosteroids remain the mainstay of treatment, ${ }^{2}$ with remission being achieved in $60 \%$ of patients treated with glucocorticoids alone. ${ }^{3,5,28,29}$
Immunomodulatory drugs, such as Methotrexate and mycophenolatemofetil, are believed to be of some benefit. $3,5,28,29$ Indications for surgery and intervention include hypertension with moderate AR, critical renal artery stenosis, extremity claudication limiting activities of daily living, cerebrovascular ischaemia or critical stenoses of three or more cerebral vessels, and cardiac ischaemia with confirmed coronary artery involvement. ${ }^{3}$

As TA is a systemic vasculopathy that can progress to cause vital organ ischaemia, long term follow up is recommended. ${ }^{2}$ Our patient has been on regular follow up and is scheduled to undergo aortic root reconstruction surgery $+/$ - stenting of the stenoses after peripheral angiography.

Although most patients show improvement with high doses of corticosteroids and immunosuppressive agents, relapses are common with tapering steroid therapy. Additionally, the pharmacological therapy only addresses one important aspect of the care of TA. ${ }^{15}$ The anatomic problems of the vascular lesions, will not respond to steroids.

As such, it has been advised that whenever feasible, correction of clinically significant lesions should be considered, especially in the setting of hypertension and severe AR among others. In about $20 \%$ of patients, aortic root involvement may lead to valvular insufficiency, angina, and congestive cardiac failure. Furthermore, patients with TA may have signs of clinical deterioration caused by fixed critical stenosis or aneurysms, 15 thus warranting surgical correction of the lesions.

Valve-sparing aortic root surgery or the Bentalloperation are effective surgical procedures, but it has been advised that the timing of the procedure must be carefully evaluated. ${ }^{30}$ It is also important to control the disease activity prior to revascularisation. Despite the advent of intervention, reviews suggest that open surgery, at present, has better outcomes compared to endovascular techniques. $^{31}$

\section{Conclusion}

TA has a myriad of presentations, and its pre-pulseless phase may be easily missed. It is also an important, albeit rare differential diagnosis to be considered in the case of early-onset hypertension with AR and fever of unknown origin, especially in young women.

\section{References:}

1. Allen HD, Driscoll DJ, Shaddy RE, Feltes TF. Diseases of the Endocardium, Myocardium, and Pericardium. Takayasu Arteritis. In: Moss and Adams' Heart Disease in 
Infants, Children and Adolescents: Including the Fetus and Young Adult. Wolters - Kluwer health - Lippincott Williams \& Wilkins; 2008, p 1288-1289.

2. Johnston SL, Lock RJ, Gompels MM. Takayasu arteritis: a review. J ClinPathol2002; 55(7): 481-486.

3. Kerr GS, Hallahan CW, Giordano J, Leavitt RY, Fauci AS, Rottem M, Hoffman GS. Takayasu arteritis. Ann Intern Med 1994; 120(11): 919-929.

4. Subramanyan R, Joy J, Balakrishnan KG. Natural history of aortoarteritis (Takayasu's disease). Circulation 1989; 80(3): 429-437.

5. Hall S, Barr W, Lie JT, Stanson AW, Kazmier FJ, Hunder GG. Takayasu arteritis. A study of 32 North American patients. Medicine (Baltimore) 1985; 64(2): 89-99.

6. Moriwaki R, Noda M, Yajima M, Sharma BK, Numano F Clinical manifestations of Takayasu arteritis in India and Japan-new classification of angiographic findings. Angiology 1997; 48(5):369-379.

7. Jain S, Sharma N, Singh S, Bali HK, Kumar L, Sharma BK. Takayasu arteritis in children and young indians. Int $\mathrm{J}$ Cardiol 2000; 75 Suppl 1:S153-157.

8. Strachan RW. Pre-pulseless and pulseless Takayasus arteritis. Postgrad Med J 1966; 42: 464-468.

9. Strachan RW. The Natural History of Takayasu's Arteriopathy. Q J Med 1964 ;33: 7-69.

10. McCulloch M, Andronikou S, Goddard E, Sinclair P, Lawrenson J, Mandelstam S, Beningfield SJ, Millar AJ. Angiographic features of 26 children with Takayasu's arteritis. PediatrRadiol 2003; 33: 230-235.

11. Morales E, Pineda C, Martinez-Lavin M. Takayasu Arteritis in children. J Rheumatol 1991; 18: 1081-1084.

12. Raju BS. Clinical Methods in Cardiology. Hyderabad: Orient Longman 2006; p206-227.

13. Abrams J. The Normal Cardiovascular Physical Examination. In: Roldan CA, Abrams J, editors. Evaluation of the patient with heart disease: Integrating the Physical Exam \& Echocardiography. Philadelphia: Lippincott Williams \& Wilkins; 2002. p1-34.

14. Schrire V, Asherson RA. Arteritis of the aorta and its major branches. Q J Med 1964; 33: 439-463.

15. Villa-Forte A, Mandell BF. Rheumatic Diseases and the Cardiovascular System. Takayasu Arteritis. In: Bonow OR, Mann DL, Zipes DP, Libby P, editors. Braunwald's Heart Disease: A Textbook of Heart Disease. 9th ed. Elsevier Saunders; 2012. p1876-78

16. Numano F, Okawara M, Inomata H, Kobayashi Y. Takayasu's arteritis. Lancet 2000; 356(9234): 1023-1025.

17. Brunner J, Feldman BM, Tyrrell PN, Kuemmerle-Deschner JB, Zimmerhackl LB, Gassner I, Benseler SM. Takayasu arteritis in children and adolescents. Rheumatology (Oxford) 2010; 49(10): 1806-1814.

18. Erten N, Saka B, Karan MA, Tascoglu C. Takayasu arteritis presenting with Fever of unknown origin: two case reports. J ClinRheumatol 2004; 10(1): 16-20.
19. Arend WP, Michel BA, Bloch DA, Hunder GG, Calabrese LH, Edworthy SM, Fauci AS, Leavitt RY, Lie JT, Lightfoot RW Jr .The American College of Rheumatology 1990 criteria for the classification of Takayasu arteritis. Arthritis Rheum 1990; 33(8): 1129-34.

20. Nazmul-Ahasan HAM, Alam B, Chowdhury MH, Mohammed FR, Nur Z. Takayasu's arteritis in association with tuberculosis in a young woman. Pak J Med Sci 2009; 25(6): 1009-1011.

21. Yotsuyanagi H, Chikatsu N, Kaneko Y, Kurokawa K. Takayasu's arteritis in prepulseless stage manifesting lymph node swelling and hepatosplenomegaly. Intern Med. 1995; 34(5): 455-459.

22. The Center for Disease Control (USA). Guidelines for Using the QuantiFERON®-TB Gold Test for Detecting Mycobacterium tuberculosis Infection, United States. CDC Expert Consultation on QuantiFERON ${ }^{\circledR}-\mathrm{TB}$ Gold. MMWR Recommendations and Reports[Internet]. Atlanta (USA): U.S. Department of Health and Human Services, The Centre for Disease Control; 2005. MMWR 2005;54(No. RR-15):1-47. [cited on 28 March 2015]. Available from : http://www.cdc.gov/mmwr/preview/ mmwrhtml/rr5415a4.htm

23. Kerr GS, Hallahan CW, Giordano J, Leavitt RY, Fauci AS, Rottem M, Hoffman GS. Takayasu arteritis. Ann Intern Med 1994; 120: 919-29.

24. Ishikawa K. Natural history and classification of occlusive thromboaortopathy (Takayasu's disease). Circulation 1978; 57: 27-35.

25. Hoffman GS, Ahmed AE. Surrogate markers of disease activity in patients with Takayasu arteritis. A preliminary report from The International Network for the Study of the Systemic Vasculitides (INSSYS). Int J Cardiol 1998; 66 Suppl 1: S191-4.

26. Devi SU. Comprehensive Cardiology. $1^{\text {st }}$ ed. New delhi: Jaypee; 2008.p 1-11Hoffman GS. Treatment of resistant Takayasu's arteritis. Rheum Dis Clin North Am 1995; 21:73-80.

27. Muranjan MN, Bavdekar SB, More V, Deshmukh H, Tripathi M, VaswaniR.Study of Takayasu's arteritis in children: clinical profile and management.J Postgrad Med. 2000;46(1):3-8.)

28. Hoffman GS. Treatment of resistant Takayasu's arteritis. Rheum Dis Clin North Am 1995; 21:73-80.

29. Hoffman GS. Takayasu arteritis: lessons from the American National Institutes of Health experience. Int J Cardiol 1996; 54 Suppl: S99-102.

30. Numano F. Vascular Manifestations in Takayasu's Arteritis. In: Ronald A, Asherson RA, Cervera R, Triplett DA, Abramson SB, editors. Vascular Manifestations of Systemic Autoimmune Diseases. Florida: Taylor and Francis Group; 2010. p252- 272.

31. Perera AH, Mason JC, Wolfe JH. Takayasu arteritis: criteria for surgical intervention should not be ignored. Int J Vasc Med 2013;2013:618910. 